\author{
Review began 10/11/2021 \\ Review ended 11/30/2021 \\ Published 12/15/2021 \\ (๑) Copyright 2021 \\ Shrestha et al. This is an open access \\ article distributed under the terms of the \\ Creative Commons Attribution License CC- \\ BY 4.0., which permits unrestricted use, \\ distribution, and reproduction in any \\ medium, provided the original author and \\ source are credited.
}

\section{Belimumab in Lupus Nephritis: A Systematic Review and Meta-Analysis}

Sanjeev Shrestha ${ }^{1}$, Pravash Budhathoki ${ }^{2}$, Yuvraj Adhikari ${ }^{3}$, Anupama Marasini ${ }^{3}$, Shakar Bhandari ${ }^{3}$, Wasey Ali Yadullahi Mir ${ }^{4}$, Dhan B. Shrestha ${ }^{4}$

1. Department of Internal Medicine, Geisinger Health System, Danville, USA 2. Department of Internal Medicine, BronxCare Health System, Bronx, USA 3. Medicine, Nepalese Army Institute of Health Sciences, Kathmandu, NPL 4. Department of Internal Medicine, Mount Sinai Hospital, Chicago, USA

Corresponding author: Dhan B. Shrestha, medhan75@gmail.com

\begin{abstract}
Belimumab is a recombinant human IgG- $1 \lambda$ monoclonal antibody. It inhibits the B-cell activating factor (BAFF) and is approved for patients with systemic lupus erythematosus (SLE) older than five years with positive autoantibody. We aimed to evaluate the role of belimumab in the maintenance phase of treatment for lupus nephritis (LN). PubMed, PubMed Central (PMC), Cochrane Library, and Embase were searched using appropriate keywords. The screening of title and abstract was done in Covidence, followed by data extraction of the relevant studies based on inclusion criteria. Review manager (RevMan 5.4) was used for data analysis with random or fixed effects model based on heterogeneities. Two randomized controlled trials were included in the quantitative analysis. There were 1.71 times higher odds of complete renal response in the belimumab group than in the control group (odds ratio (OR), 1.71; 95\% confidence interval (CI), 1.12-2.60; I-square $\left(\mathrm{I}^{2}\right)=0 \%$ ). Similarly, there was $34 \%$ lower odds for having no response among the belimumab group (OR, 0.66; 95\% CI, 0.45-0.96; $\mathrm{I}^{2}=0 \%$ ). No significant differences between the two groups were observed for the occurrence of treatment-related adverse events (TRAEs) (OR, 1.07; 95\% CI, 0.74-1.56; $\mathrm{I}^{2}=0 \%$ ), treatment-related serious adverse events (OR, 0.54; 95\% CI, 0.15-1.96; $\mathrm{I}^{2}=68 \%$ ), and treatmentrelated infections $\left(\mathrm{OR}, 0.65 ; 95 \% \mathrm{CI}, 0.27-1.55 ; \mathrm{I}^{2}=21 \%\right)$.Therefore, belimumab and standard treatment were instrumental for beneficial renal response in patients with lupus nephritis and were not associated with increased odds of adverse effect compared with the standard treatment alone.
\end{abstract}

Categories: Allergy/Immunology, Nephrology, Rheumatology

Keywords: monoclonal antibody, meta-analysis, systemic lupus erythematosus, lupus nephritis, belimumab

\section{Introduction And Background}

Systemic lupus erythematosus (SLE) is a chronic inflammatory disease with variable clinical manifestations. Lupus nephritis (LN) is one of the most common and serious manifestations of SLE. Lupus activity involving the kidney is characterized by nephrotic range proteinuria and hematuria with lupus stage-specific immunological findings. The symptoms of LN vary with stage; however, proteinuria, hypertension, and later, renal failure occur in advanced disease. $\mathrm{LN}$ of clinical relevance is diagnosed when the creatinine clearance decrease by $30 \%$, with proteinuria of $>500 \mathrm{mg} / \mathrm{dL}$, and renal biopsy histological findings support active LN [1]. It occurs in nearly $50 \%$ of patients with SLE but is not the only cause of kidney injury in SLE [2]. LN treatment consists of an induction phase and a maintenance phase. In the induction phase, combination therapy with an immunosuppressive agent including steroids, cyclophosphamide (CYC), or mycophenolate mofetil (MMF) is employed to induce immune quiescence and reduce the ongoing renal inflammation [3]. Induction is intended for a complete renal response and to reduce early damage, preserving renal function in the long run. The maintenance phase is intended to avoid renal flares, minimizing glucocorticoid exposure and associated toxicity due to the use of immunosuppressants. The present treatment regimens for $\mathrm{LN}$ are associated with high drug-associated toxicity and low treatment efficacy and adherence [3]. In this context, belimumab has emerged as one of the emerging drugs for treating lupus nephritis. Belimumab is a recombinant IgG- $1 \lambda$ monoclonal antibody that acts by inhibiting B-cell activation and is approved for patients with SLE aged greater than five years with autoantibody activity [4]. Belimumab targets soluble B-cell activating factor (BAFF) and possibly helps prevent the development of autoreactive B-cells [3]. The most common side effects of belimumab are nausea, diarrhea, pyrexia, nasopharyngitis, etc. We conducted this study to assess the efficacy and safety of belimumab in patients with lupus nephritis.

\section{Review}

\section{Methods}

We followed the guidelines of the Preferred Reporting Items for Systematic Reviews and Meta-Analyses (PRISMA) to conduct this meta-analysis [5]. 


\section{Cureus}

\section{Study protocol}

An initial search and review of the literature were done on research questions. As a result, the protocol was published in Prospero (CRD420212340448) on February 10, 2021 [6].

\section{Information sources}

Electronic databases such as PubMed Central (PMC), PubMed, Cochrane Library, and Embase were searched for relevant articles with keywords such as "belimumab" and "lupus nephritis."

\section{Study records}

Data Management

All the articles that met the keyword were uploaded into the Mendeley Reference Manager software, where first screening for duplicates was carried out, and those files were imported into Covidence to check for any duplicates and formal screening.

Selection Process

Two of our authors acted as independent reviewers (SB and YA) and screened the articles independently based on title and abstract. Then, any conflicts that arose during the screening were resolved by the third reviewer (AM). Two reviewers (YA and AM) did full-text reviews independently, and the third reviewer (SB) reviewed and resolved disputes in the Covidence screening software based on the preset inclusion and exclusion criteria.

All comparative studies (RCTs, cohort studies, case-control studies, cross-sectional studies, etc.) comparing belimumab with placebo and other standard care in lupus nephritis were included in our study. Editorials, commentaries, viewpoints, and studies with no proper data regarding efficacy, safety between belimumab with Placebo, and other standard care in lupus nephritis were excluded.

Data Collection Process

Necessary data were collected from selected studies using a predesigned Population Intervention Comparison and Outcome (PICO) formatted Microsoft Excel form and checked by all three reviewers for accuracy. The data extraction form included study details such as study ID, year of the study, study population characteristics (e.g., total number, age, sex, and other relevant disease-specific parameters such as comorbidities), intervention (dose and duration of the study drug), comparator (placebo or standard care), and outcome (changes in base value, adverse effect, and mortality).

\section{Outcome}

The primary outcomes were efficacy in terms of good renal response and safety of belimumab in lupus nephritis.

\section{Risk of bias in individual studies}

The individual articles were evaluated using Cochrane risk-of-bias tool for randomized trials (RoB 2.0) for their methodological quality (Figure 1) [7]. 


\section{Cureus}

\begin{tabular}{|c|c|c|c|c|c|c|c|}
\hline $\begin{array}{l}\text { High risk } \\
\begin{array}{|l|}+ \\
\text { Low risk } \\
? \\
\text { Unclear } \\
\text { risk }\end{array}\end{array}$ & 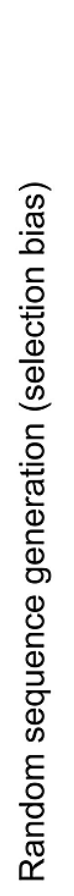 & 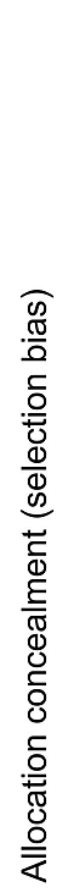 & 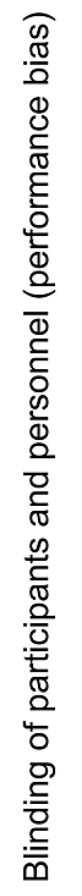 & 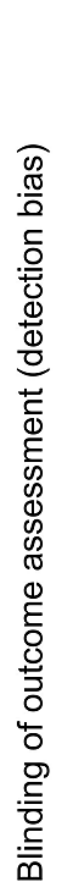 & 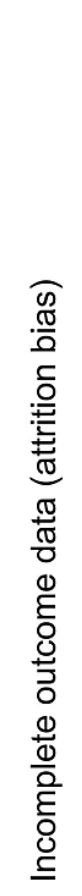 & 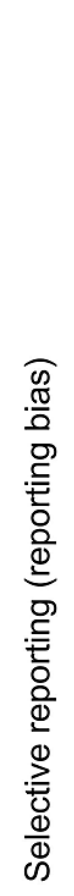 & 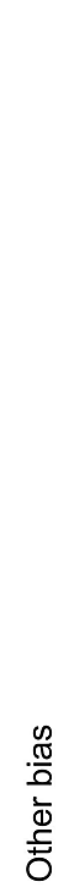 \\
\hline sha-Fregoso Y, et al. 2021 & + & $?$ & & & + & + & $?$ \\
\hline Furie R, et al. 2020 & + & + & + & + & + & & $?$ \\
\hline
\end{tabular}

FIGURE 1: Risk-of-bias summary of randomized controlled trials

\section{Data synthesis}

RevMan 5.4 was used for the analysis of the extracted data. Based on the heterogeneities across the studies, a random/fixed effects model was used.

\section{Assessment of heterogeneity}

Assessment of heterogeneity was done using I-square $\left(\mathrm{I}^{2}\right)$ test based on the Cochrane Handbook for Systematic review [8].

\section{Sensitivity analysis}

Sensitivity analysis was not performed due to the paucity of studies regarding the use of belimumab in lupus nephritis.

\section{Result}

A total of 1728 studies were identified after thorough database searching, and duplicates were removed. The titles and abstracts of 1273 studies were screened, and 1257 studies were excluded. In addition, 11 studies were assessed for full-text eligibility. Nine studies were excluded for definite reasons, and two studies were 


\section{Cureus}

included in the qualitative and quantitative analysis (Tables 1,2). The following information is presented on the PRISMA flow diagram (Figure 2).

\begin{tabular}{|c|c|c|c|}
\hline ID & Country & Inclusion criteria & Exclusion criteria \\
\hline $\begin{array}{l}\text { Atisha- } \\
\text { Fregoso } \\
\text { et al., } \\
2021[3]\end{array}$ & $\begin{array}{l}\text { United } \\
\text { States }\end{array}$ & $\begin{array}{l}\text { 1. Age }>18 \text { years, diagnosis fulfilling the ACR or SLICC criteria for } \\
\text { SLE 2. ANAs and/or anti-double-stranded DNA (anti-dsDNA) } \\
\text { antibodies positive at the time of screening } 3 \text {. Recurrent or refractory } \\
\text { LN treated with either CYC or MMF previously }\end{array}$ & $\begin{array}{l}\text { Already a case treated with rituximab or } \\
\text { another B-cell biologic therapy within the } \\
\text { prior } 12 \text { months }\end{array}$ \\
\hline $\begin{array}{l}\text { Furle et } \\
\text { al., } \\
2020[4]\end{array}$ & $\begin{array}{l}\text { Multinational } \\
(21 \\
\text { countries, } \\
107 \text { sites) }\end{array}$ & $\begin{array}{l}\text { 1. Age } \geq 18 \text { years; autoantibody-positive } 2 \text {. SLE (ANA titers } \geq 1: 80 \text {, } \\
\text { anti-dsDNA antibodies, or both); urinary protein/creatinine } \geq 1 \text { and } \\
\text { biopsy-proven LN ISNRPS class III (focal lupus nephritis) or IV } \\
\text { (diffuse lupus nephritis) with or without coexisting class } V \\
\text { (membranous lupus nephritis) or pure class } V \text { lupus nephritis within } \\
\text { six months before or during screening } 3 \text {. Patients with active lesions } \\
\text { or active and chronic lesions in biopsy }\end{array}$ & $\begin{array}{l}\mathrm{mL} / \mathrm{minute} / 1.73 \mathrm{~m}^{2} \text { of body surface area; } \\
\text { previous failure with either CYC or MMF } \\
\text { induction; induction with CYP within three } \\
\text { months before the trial; and B-cell- } \\
\text { targeted therapy (including belimumab) } \\
\text { one year before randomization }\end{array}$ \\
\hline
\end{tabular}

\section{TABLE 1: Baseline details of the included studies}

ANAs: antinuclear antibodies; DNA: deoxyribonucleic acid; SLE: systemic lupus erythematosus; MMF: mycophenolate mofetil; CYC: cyclophosphamide; LN: lupus nephritis; ACR: American College of Rheumatology; eGFR: estimated glomerular filtration rate; SLICC: Systemic Lupus International Collaborating Clinics; ISNRPS: International Society of Nephrology and Renal Pathology Society

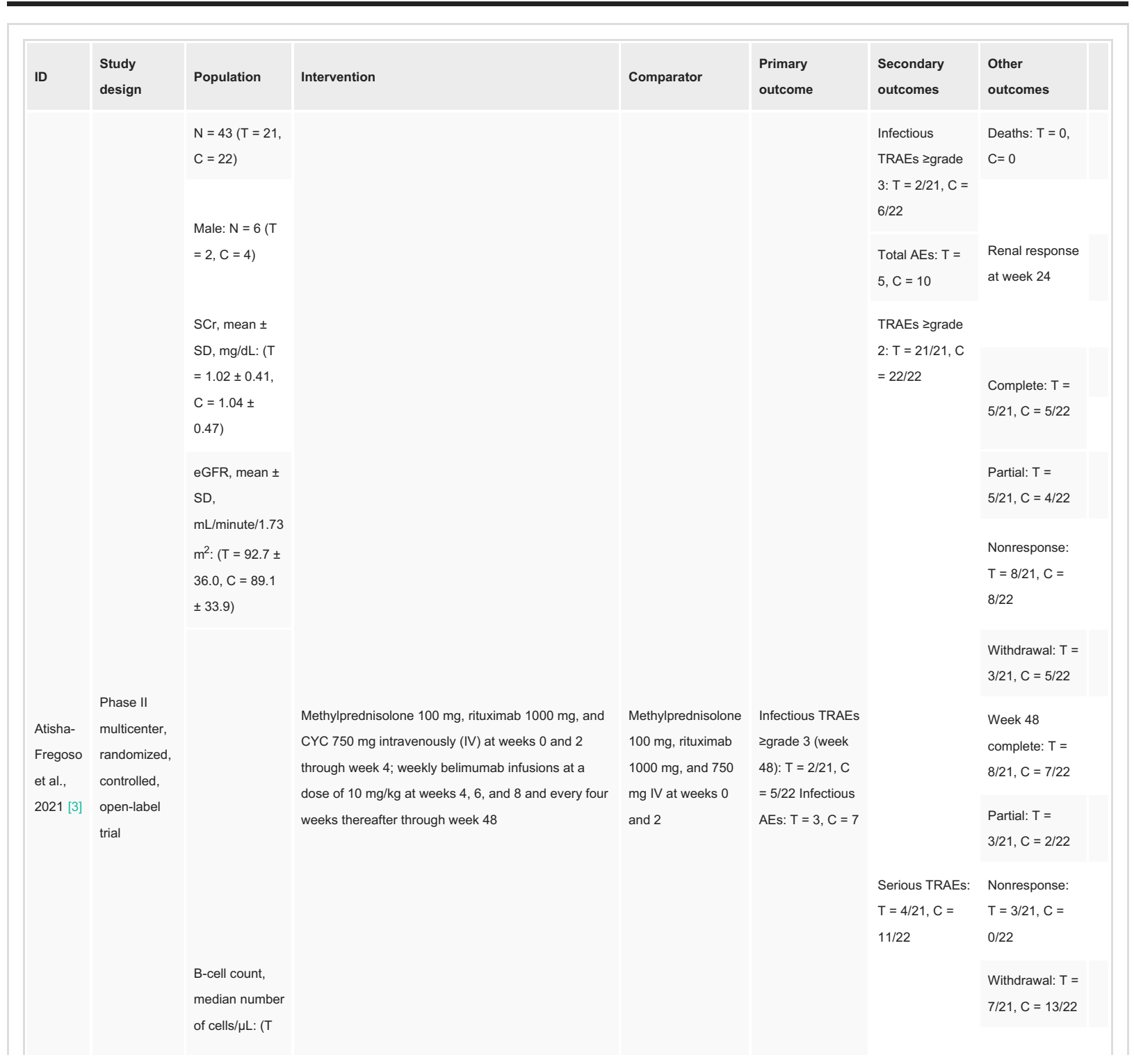




\section{Cureus}

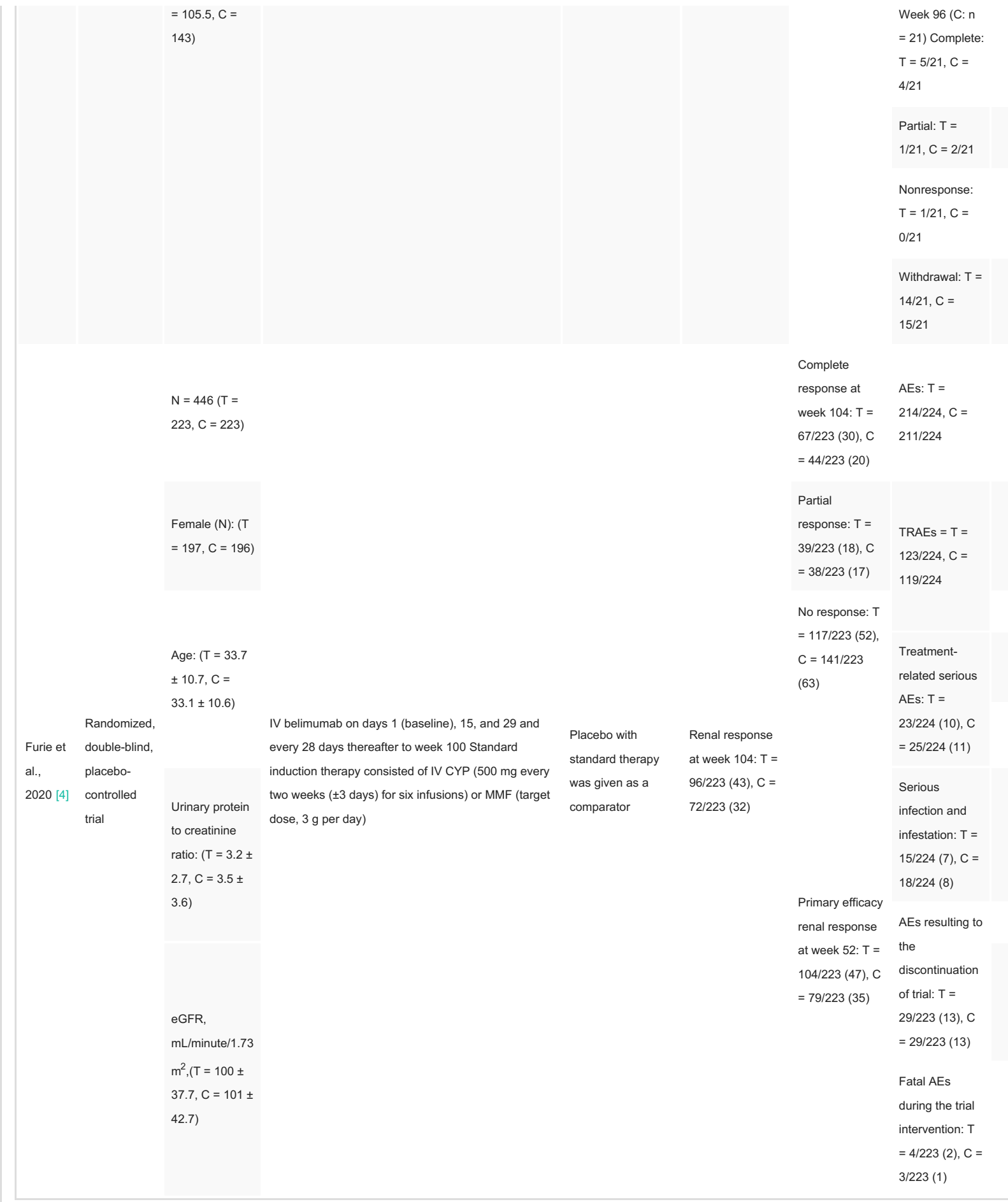

\section{TABLE 2: Qualitative synthesis of the included studies}

T: treatment; TRAEs: treatment-emergent adverse events; C: control; N: total number of participants; SD: standard deviation; CYC: cyclophosphamide; eGFR: estimated glomerular filtration rate; IV: intravenous; AEs: adverse events 


\section{Cureus}
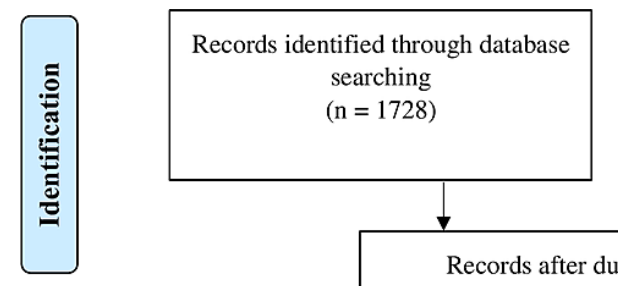

Additional records identified through other sources $(n=0)$

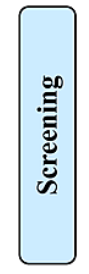

Records after duplicates removed $(\mathrm{n}=1273)$

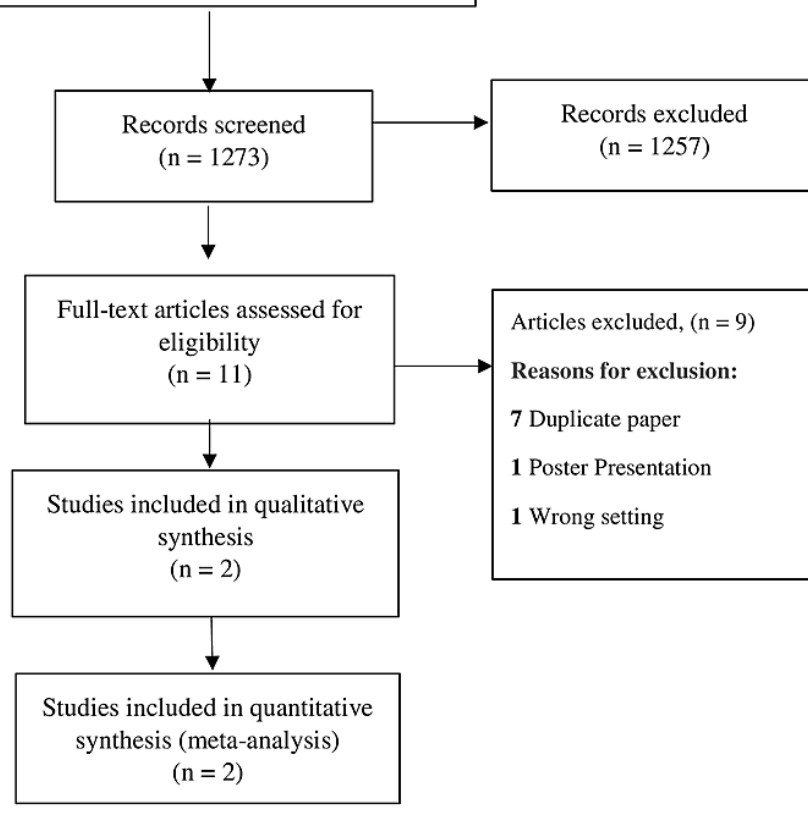

FIGURE 2: PRISMA flow diagram

Efficacy of Belimumab in Lupus Nephritis

The pooling data of two studies $(n=488)$ showed that there were 1.71 times higher odds of complete renal response in the belimumab group than in the control group (odds ratio (OR), 1.71; $95 \%$ confidence interval (CI), 1.12-2.60; $\mathrm{I}^{2}=0 \%$ ). Similarly, there was $34 \%$ lower odds for having no response among the belimumab group (OR, 0.66; 95\% CI, 0.45-0.96; $\mathrm{I}^{2}=0 \%$ ). However, there was no difference between the two groups regarding partial renal response $\left(\mathrm{OR}, 1.00 ; 95 \% \mathrm{CI}, 0.62-1.62 ; \mathrm{I}^{2}=0 \%\right)$ (Figure 3). 


\section{Cureus}

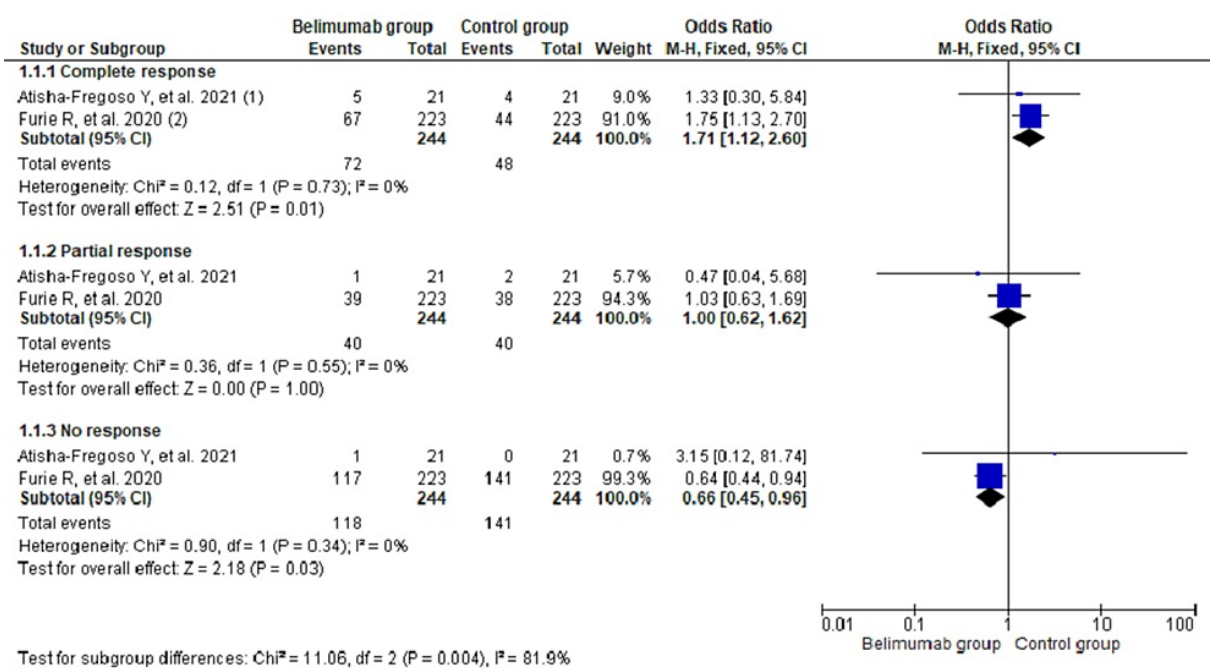

Test for subgroup differences: $\mathrm{Ch}^{2}=11.06, \mathrm{df}=2(P=0.004), I^{2}=81.9 \%$

$\frac{\text { Footnotes }}{\text { (1) Atweek }}$

(1)Alweek 90

FIGURE 3: Forest plot showing renal response among the two groups of treatment recipient patients with lupus nephritis using fixed effects model

$\mathrm{Cl}$ : confidence interval

Safety of Belimumab in Lupus Nephritis

The pooling data on adverse events during treatment using random effects model showed no significant differences between two groups for the occurrence of treatment-related adverse events (TRAEs) (OR, 1.07; $95 \% \mathrm{CI}, 0.74-1.56 ; \mathrm{I}^{2}=0 \%$ ), treatment-related serious adverse events (OR, 0.54; $95 \% \mathrm{CI}, 0.15-1.96 ; \mathrm{I}^{2}=68 \%$ ), and treatment-related infections (OR, 0.65; 95\% CI, 0.27-1.55; $\mathrm{I}^{2}=21 \%$ ) (Figure 4).

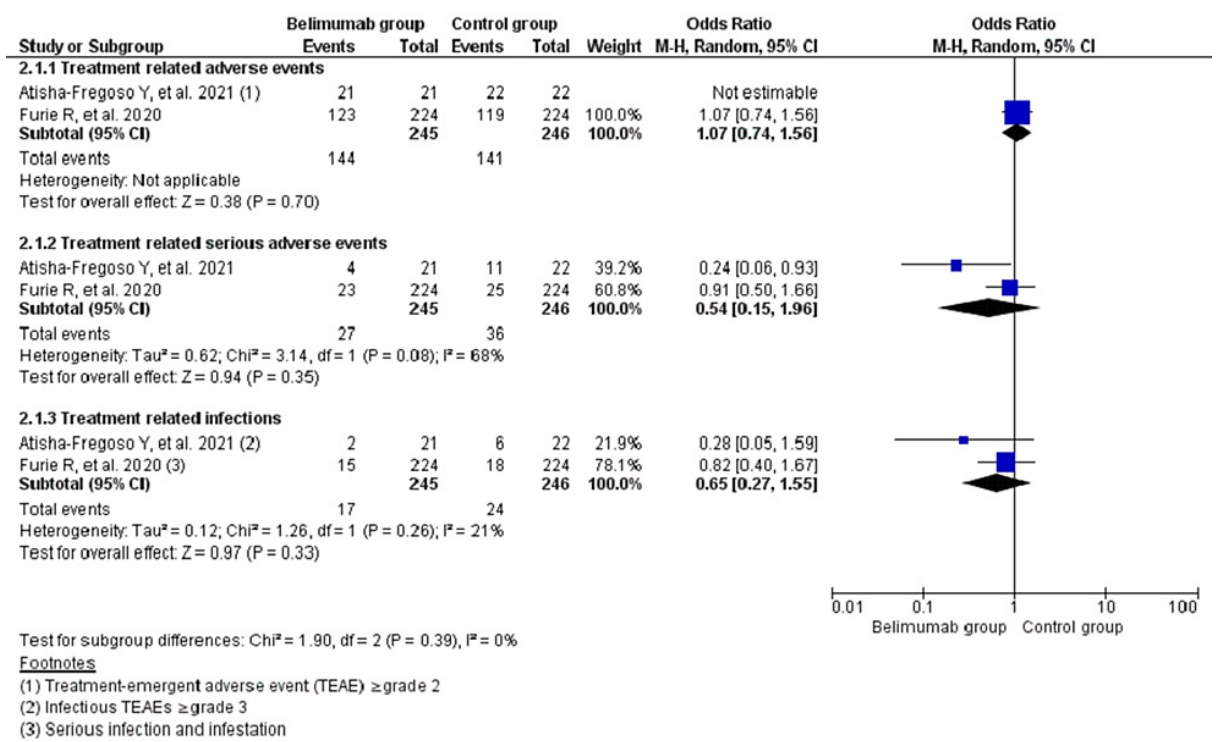

FIGURE 4: Forest plot showing treatment-related adverse events among the two groups of treatment recipient patients with lupus nephritis using a random effects model

Cl: confidence interval 


\section{Cureus}

\section{Discussion}

The meta-analysis showed higher odds of renal response in patients receiving belimumab compared with patients receiving standard of treatment and no significant difference in adverse effect between belimumab and standard treatment. These findings are significant because higher risks of relapse and poor term outcomes are typically observed in lupus nephritis [9-11]. The common findings in patients with lupus nephritis are increased production of BAFF in the kidney, leading to increased levels of BAFF in the blood [12-14]. To manage lupus nephritis, it is imperative to neutralize the B-cell activating factor to decrease Bcell function, autoantibody production, and suppression of lymphoid structure formation in the kidney. Belimumab has been shown to produce partial depletion of B-cells and cause low circulating levels of BAFF [15]. This might explain better renal response seen in patients with lupus nephritis who received belimumab in addition to usual standard medications compared with standard of treatment alone. The prevention of renal flare is instrumental in the prevention of worse outcomes seen with lupus nephritis. The odds for the adverse event were similar in a patient receiving belimumab in addition to standard treatment compared with standard treatment alone. A similar finding was seen in the Rituximab and Belimumab for Lupus Nephritis (CALIBRATE) trial in which belimumab was found to be a safe treatment in lupus nephritis [3]. The safety of belimumab in the treatment of SLE has already been well established, and a prior meta-analysis done by Wei et al. found no significant difference in the adverse event of belimumab in SLE [16].

Our meta-analysis is the first meta-analysis to evaluate the efficacy and safety of belimumab in lupus nephritis performed after a comprehensive search of various databases. However, our study has a few limitations. There was a lack of an adequate number of studies in our analysis. The included studies had their inherent limitations, such as low enrollment of Black patients and patients receiving cyclophosphamide and azathioprine, as well as lack of patient-reported outcomes in the Belimumab in Subjects With Systemic Lupus Erythematosus (BLISS) trial [4]. Similarly, the CALIBRATE trial was underpowered and included patients with only refractory or recurrent lupus nephritis [3].

\section{Conclusions}

Belimumab, a monoclonal antibody, is a newer treatment option for SLE in reducing the side effect of immunomodulatory drugs. Belimumab has shown promising results in patients with lupus. Its addition to standard treatment showed an improved renal response in patients with lupus nephritis and was not associated with increased odds of adverse effects compared with the standard treatment alone. Due to the paucity of studies among patients with lupus nephritis, further study in this subgroup of patients with lupus is recommended to affirm these initial promising findings.

\section{Appendices}

\section{Belimumab and lupus nephritis electronic search details}

PubMed

Keywords: "belimumab" and "lupus nephritis"

Hits: 124

Link: https://pubmed.ncbi.nlm.nih.gov/?term=belimumab+and+lupus+nephritis

PubMed Central

Keywords: "belimumab" and "lupus nephritis"

Hits: 787

Link: https://www.ncbi.nlm.nih.gov/pmc/?term=\%22belimumab\%22+and+\%22lupus+nephritis\%22

Cochrane Library

Keywords: "belimumab" in All Text AND "lupus nephritis" in All Text

Hits: 20

Link: https://www.cochranelibrary.com/advanced-search

Embase

Keywords: ("belimumab"/exp OR belimumab) AND (“lupus nephritis"/exp OR "lupus nephritis") 


\section{Additional Information \\ Disclosures}

Conflicts of interest: In compliance with the ICMJE uniform disclosure form, all authors declare the following: Payment/services info: All authors have declared that no financial support was received from any organization for the submitted work. Financial relationships: All authors have declared that they have no financial relationships at present or within the previous three years with any organizations that might have an interest in the submitted work. Other relationships: All authors have declared that there are no other relationships or activities that could appear to have influenced the submitted work.

\section{References}

1. Farah RI, Dannoun E, Abu Shahin N, AlRyalat SA: Characteristics and histological types of lupus nephritis in a Jordanian tertiary medical center. Biomed Res Int. 2019, 2019:7087461. 10.1155/2019/7087461

2. Parikh SV, Almaani S, Brodsky S, Rovin BH: Update on lupus nephritis: core curriculum 2020 . Am J Kidney Dis. 2020, 76:265-81. 10.1053/j.ajkd.2019.10.017

3. Atisha-Fregoso Y, Malkiel S, Harris KM, et al.: Phase II randomized trial of rituximab plus cyclophosphamide followed by belimumab for the treatment of lupus nephritis. Arthritis Rheumatol. 2021, 73:121-31. 10.1002/art.41466

4. Furie R, Rovin BH, Houssiau F, et al.: Two-year, randomized, controlled trial of belimumab in lupus nephritis. N Engl J Med. 2020, 383:1117-28. 10.1056/NEJMoa2001180

5. Liberati A, Altman DG, Tetzlaff J, et al.: The PRISMA statement for reporting systematic reviews and metaanalyses of studies that evaluate healthcare interventions: explanation and elaboration. BMJ. 2009, 339:b2700. 10.1136/bmj.b2700

6. Belimumab in lupus nephritis: a systematic review and meta-analysis . (2021). Accessed: April 21, 2021: https://www.crd.york.ac.uk/prospero/display_record.php?RecordID=230448.

7. Sterne JA, Savović J, Page MJ, et al.: RoB 2: a revised tool for assessing risk of bias in randomised trials . BMJ. 2019, 366:14898. 10.1136/bmj.14898

8. Cochrane handbook for systematic reviews of interventions. Higgins JPT, Thomas J, Chandler J, Cumpston M, Li T, Page MJ, Welch VA (ed): John Wiley \& Sons, Chichester, UK; 2019.

9. Tamirou F, D'Cruz D, Sangle S, et al.: Long-term follow-up of the MAINTAIN Nephritis Trial, comparing azathioprine and mycophenolate mofetil as maintenance therapy of lupus nephritis. Ann Rheum Dis. 2016, 75:526-31. 10.1136/annrheumdis-2014-206897

10. Dall'Era M, Cisternas MG, Smilek DE, et al.: Predictors of long-term renal outcome in lupus nephritis trials: lessons learned from the Euro-Lupus Nephritis cohort. Arthritis Rheumatol. 2015, 67:1305-13. 10.1002/art.39026

11. Davidson JE, Fu Q, Ji B, Rao S, Roth D, Magder LS, Petri M: Renal remission status and longterm renal survival in patients with lupus nephritis: a retrospective cohort analysis. J Rheumatol. 2018, 45:671-7. 10.3899/jrheum.161554

12. Schwarting A, Relle M, Meineck M, et al.: Renal tubular epithelial cell-derived BAFF expression mediates kidney damage and correlates with activity of proliferative lupus nephritis in mouse and men. Lupus. 2018, 27:243-56. 10.1177/0961203317717083

13. Sun CY, Shen Y, Chen XW, et al.: The characteristics and significance of locally infiltrating B cells in lupus nephritis and their association with local BAFF expression. Int J Rheumatol. 2013, 2013:954292. $10.1155 / 2013 / 954292$

14. Kang S, Fedoriw Y, Brenneman EK, Truong YK, Kikly K, Vilen BJ: BAFF induces tertiary lymphoid structures and positions T cells within the glomeruli during lupus nephritis. J Immunol. 2017, 198:2602-11. 10.4049/jimmunol.1600281

15. Jacobi AM, Huang W, Wang T, et al.: Effect of long-term belimumab treatment on B cells in systemic lupus erythematosus: extension of a phase II, double-blind, placebo-controlled, dose-ranging study. Arthritis Rheum. 2010, 62:201-10. 10.1002/art.27189

16. Wei LQ, Liang YG, Zhao Y, Liang HT, Qin DC, She MC: Efficacy and safety of belimumab plus standard therapy in patients with systemic lupus erythematosus: a meta-analysis. Clin Ther. 2016, 38:1134-40. 10.1016/j.clinthera.2016.02.022 